\title{
Incidencia y manejo del gusher en la implantación coclear
} Incidence and management of gusher in cochlear implantation

\author{
Antonio Soda-Merhy*, Gary J. Olguín-Vega, Lisette Cristerna-Sánchez y Fernándo Martín-Biasotti \\ Departamento de Otorrinolaringología, Instituto Nacional de Enfermedades Respiratorias ICV, Ciudad de México, México
}

\section{Resumen}

Objetivo: Presentar un reporte de la incidencia y del protocolo de manejo del gusher del líquido cefalorraquídeo durante la implantación coclear en el Instituto Nacional de Enfermedades Respiratorias. Método: Se realizó una investigación clínica, longitudinal y retrospectiva de los expedientes clínicos de todos los pacientes con implantación coclear entre octubre de 1999 y diciembre de 2016, para valorar la incidencia de Gusher, el protocolo de manejo y la evolución posterior. Se realizó el análisis estadístico utilizando la prueba exacta de Fisher para buscar si había relación entre el número de malformaciones de oído interno y el grado de Gusher, y entre la vía de abordaje al oído interno (cocleostomía o ventana redonda) y el grado de Gusher. Resultados: Se valoraron 276 pacientes, de los cuales 12 (4.3\%) presentaron Gusher durante la implantación coclear. De estos, siete fueron Gusher mínimo y cedió totalmente con el sellado con fascia, tres fueron Gusher moderado que ameritó el sellado con fascia y cartílago, y dos fueron Gusher grave que ameritó empaquetamiento de oído medio y obliteración de la trompa de Eustaquio. No se encontró una relación estadísticamente significativa entre el número de malformaciones del oído interno o la vía de abordaje al oído interno con el grado de Gusher. Conclusiones: Debe realizarse una minuciosa evaluación del estudio de imagen prequirúrgico, que incluya la toma de mediciones específicas, y brindar un manejo individualizado según la gravedad del Gusher.

PALABRAS CLAVE: Acueducto vestibular ensanchado. Gusher. Líquido cefalorraquídeo. Partitura incompleta. Oozing.

\begin{abstract}
Objective: To present a report on the incidence and management protocol of cerebrospinal fluid gusher in cochlear implantation in the national institute of respiratory diseases. Method: We conducted a clinical, longitudinal, retrospective investigation of all the medical charts from implanted patients between October 1999 and December 2016 in the national institute of respiratory diseases to evaluate the incidence of gusher, management protocol and evolution after surgery. Statistical analysis was conducted using Fishers test to find out if there was a relationship between the number of inner ear malformations and gusher severity and between the surgical access to the inner ear (cochleostomy or round window) and gusher severity. Results: 276 patients were evaluated, 12 (4.3\%) presented gusher during cochlear implantation. Seven had minimal gusher that stopped completely with a fascia seal, three had moderate gusher that required fascia and cartilage and two had severe gusher that required middle ear packing and obliteration of the eustachian tube. There was no statistically significant relationship between the number of inner ear malformations or the surgical access to the inner ear with gusher severity. Conclusions: $A$ thorough evaluation of the imaging study should always be conducted, it should include specific measurements and an individualized management in accordance with gusher severity should always be performed.
\end{abstract}

KEY WORDS: Enlarged vestibular aqueduct. Gusher. Cerebrospinal fluid. Incomplete partiture. Oozing.

\author{
Correspondencia: \\ *Antonio Soda-Merhy \\ Vialidad de la Barranca S/N, 840 \\ Col. Valle de las Palmas \\ C.P. 52763, Huixquilucan, Estado de México, México \\ E-mail: asodam@ @rodigy.net.mx
}

Fecha de recepción: 23-10-2018

Fecha de aceptación: 08-02-2019

DOI: $10.24875 / C I R U .19000826$
Cir Cir. 2019;87:650-655 www.cirugiaycirujanos.com 


\section{Introducción}

El término gusher se utiliza para describir la salida profusa de líquido cefalorraquídeo (LCR) al acceder a la vuelta basal de la cóclea. Algunos autores especifican que se trata de una fuga de LCR de 10-20 minutos de duración que requiere medidas específicas para su control. Debe recordarse que, normalmente, el LCR no se encuentra presente en la cóclea, sino que más bien se encuentra en el espacio subaracnoideo, el cual se extiende hacia el conducto auditivo interno y llega casi al fundus, donde se encuentra separado del líquido perilinfático de la cóclea por una lámina ósea localizada en la porción terminal lateral del conducto auditivo interno, llamada lámina cribosa'. Teniendo esto en cuenta, se cree que el gusher ocurre debido a dos factores específicos: la presencia de una comunicación anómala entre los espacios subaracnoideo y perilinfático a través de la lámina cribosa (por una alteración en su desarrollo embriológico o por un traumatismo en el hueso temporal), y un diámetro anormal del acueducto coclear².

Es importante destacar otras características del gusher en la implantación coclear, como que se encuentra asociado a una mayor incidencia de meningitis en el posoperatorio inmediato y tardío, y que suele asociarse a malformaciones del oído interno concretas, como la partitura incompleta coclear tipo I y tipo III según la clasificación de Sennaroglu, y el síndrome del acueducto vestibular ensanchado ${ }^{3}$. Sin embargo, debe destacarse que puede presentarse incluso en pacientes con un estudio de imagen prequirúrgico completamente normal, porque también ocurre en pacientes con malformaciones modiolares menores, e incluso se ha llegado a encontrar que existe una relación entre el grado de defecto modiolar y el grado de gusher.

En la actualidad, diversos autores manejan también el término oozing, que se define como una fuga de LCR intermitente, menos grave y que no suele requerir maniobras específicas para su control. Se cree que se debe a un defecto menor entre el oído interno malformado y el conducto auditivo interno. Suele detenerse en un plazo de minutos y se asocia a partitura incompleta tipo II de Sennaroglu y al síndrome del acueducto vestibular ensanchado ${ }^{3}$.

El objetivo primario de este trabajo fue presentar un reporte de la incidencia y del protocolo de manejo del gusher durante la implantación coclear en el Instituto Nacional de Enfermedades Respiratorias, y como objetivo secundario buscar si existe una relación entre el número de malformaciones del oído interno, el abordaje al oído interno y el grado de gusher.

\section{Método}

Se realizó una investigación clínica, longitudinal y retrospectiva de los expedientes clínicos de 276 pacientes con diagnóstico de hipoacusia sensorineural de grave a profunda a quienes se colocó un implante coclear en el Instituto Nacional de Enfermedades Respiratorias entre el 7 de octubre de 1999 y el 20 de diciembre de 2016, para valorar la incidencia de gusher y su gravedad, el manejo durante el transoperatorio y el posoperatorio, y también el desempeño posquirúrgico de estos pacientes.

Los criterios de inclusión fueron pacientes de sexo y edad indistintos, que presentaron gusher durante el procedimiento quirúrgico (claramente descrito y clasificado en la nota quirúrgica) y que contaran con un expediente clínico completo. Los criterios de exclusión fueron pacientes que no presentaron gusher durante el procedimiento quirúrgico.

La clasificación de la gravedad del gusher la realizó el cirujano primario durante el acto quirúrgico, y se definió de acuerdo con las medidas que se requirieron para su control:

- Oozing: salida de LCR mínima que cedió espontáneamente.

- Mínimo: el sellado con fascia temporal detuvo la fuga por completo.

- Moderado: el sellado con fascia temporal y cartílago de trago detuvo la fuga por completo.

- Grave: además de las medidas mencionadas, se realizaron empaquetamiento del oído medio y obliteración de la trompa de Eustaquio para detener la fuga.

Así mismo, tres otorrinolaringólogos y un radiólogo entrenados en el campo de otología hicieron una revisión retrospectiva de los estudios de imagen prequirúrgicos que involucró la medición de diversas estructuras del oído interno mediante software especializado, y clasificaron las diversas malformaciones del oído interno que encontraron según la clasificación de Sennaroglu4.

La técnica quirúrgica utilizada en todos los pacientes consistió en incisión retroauricular, mastoidectomía cortical, aticotomía, timpanotomía posterior y cocleostomía anteroinferior a la ventana redonda o abordaje a través de la ventana redonda. Todos los casos que presentaron gusher fueron detectados en el momento de la cirugía primaria. 
Tabla 1. Grupo de estudio

\begin{tabular}{lllllll}
\hline Paciente & Sexo & Edad & Hallazgos en la TC & Abordaje al oído interno & Grado de gusher & Manejo \\
\hline 1 & F & 5 & LCE & Ventana redonda & MÍnimo & Fascia \\
2 & M & 3 & PI II & Cocleostomía & MÍnimo & Fascia \\
3 & M & 2 & LCE & Cocleostomía & MÍnimo & Fascia \\
4 & M & 3 & PI II y AVE & Cocleostomía & Moderado & Fascia, cartílago \\
5 & M & 1 & LCE & Cocleostomía & Mínimo & Fascia \\
6 & M & 4 & AVE & Ventana redonda & Mínimo & Fascia \\
7 & M & 12 & PI II, AVE y VD & Cocleostomía & MÍnimo & Fascia \\
8 & F & 3 & AVE & Cocleostomía & Grave & Empaquetamiento, \\
& & & PI II, AVE y VD & Ventana redonda & MÍnimo & Fascia \\
9 & F & 1 & PI I, VD y DCSL & Ventana redonda & Grave & Empaquetamiento, \\
10 & M & 5 & Obliteracion trompa de Eustaquio \\
11 & F & 2 & PI II y AVE & Ventana redonda & Moderado & Fascia, cartílago
\end{tabular}

AVE: acueducto vestibular ensanchado; DCSL: displasia del canal semicircular lateral; F: femenino; LCE: lámina cribosa ensanchada; M: masculino; PI: partitura incompleta; VD: vestíbulo dilatado; TC: tomografía computarizada.

En lo que se refiere al análisis estadístico, fue realizado con el programa Stata 13 . Se utilizó la prueba exacta de Fisher para averiguar si existía relación entre el número de malformaciones del oído interno y el grado de gusher, y entre la vía de abordaje al oído interno (cocleostomía o ventana redonda) y el grado de gusher. Se consideró como estadísticamente significativa una $p=0.05$.

\section{Resultados}

De los 276 pacientes que recibieron un implante, $12(4.3 \%)$ presentaron gusher durante el acto quirúrgico. El abordaje quirúrgico que se utilizó fue la cocleostomía en siete pacientes y por la ventana redonda en cinco pacientes. De acuerdo con la clasificación del grado de gusher previamente descrita, siete pacientes presentaron gusher mínimo, tres gusher moderado y dos gusher grave. Los siete casos de gusher mínimo cedieron totalmente con el sellado del sitio de cocleostomía con fascia temporal, como solemos realizar en todos nuestros procedimientos. Los tres casos de gusher moderado ameritaron sellado del sitio de cocleostomía con fascia temporal y un refuerzo con una segunda capa de fibrina y cartílago de trago. Los dos cuadros de gusher grave requirieron empaquetamiento del oído medio y obliteración de la trompa de Eustaquio (Tabla 1).
En lo que se refiere a la presencia de malformaciones detectables por imagen, tres pacientes presentaron una malformación única y nueve presentaron más de una malformación. Las malformaciones que se detectaron, por orden de frecuencia, fueron acueducto vestibular ensanchado (nueve pacientes), partitura incompleta tipo II (seis pacientes), vestíbulo dilatado (seis pacientes), lámina cribiforme ensanchada (cuatro pacientes) y partitura incompleta tipo I (dos pacientes).

Al realizar el análisis estadístico no se encontró una relación estadísticamente significativa entre el número de malformaciones del oído interno y el grado de gusher ( $p=0.15)$, ni tampoco entre la vía de abordaje al oído interno y el grado de gusher $(p=0.57)$.

En cuanto a los resultados funcionales, de los 12 pacientes reportados, 10 se encuentran actualmente asistiendo a una escuela para normooyentes y con un buen desempeño, e incluso llegan a responder a órdenes complejas en contexto abierto; una paciente fue explantada por motivos ajenos al gusher y un paciente presenta un bajo desempeño (este paciente presenta diversos problemas neurológicos desde el nacimiento).

\section{Discusión}

La presencia de gusher durante la implantación coclear fue descrita por primera vez por Miyamoto en 
1986 en un paciente que presentaba una malformación del oído interno tipo Mondini, como solía describirse en aquel entonces. Actualmente, diversas series reportan una incidencia en general entre el 1 y el $5 \%$. Sin embargo, en trabajos que se centran exclusivamente en pacientes con malformaciones del oído interno esta cifra suele llegar hasta un 40-50\%. Se cree que la variabilidad en las cifras sobre la incidencia de gusher en la literatura puede deberse a que se incluyen fugas mínimas de LCR, conocidas como oozing, que llevan a sobreestimar el número real de casos. Es importante destacar la relación existente entre la presencia de gusher y las malformaciones del oído interno, debido a que estas se presentan de manera visible en el estudio de imagen prequirúrgico hasta en un $20 \%$ de los pacientes con hipoacusia sensorineural de grave a profunda. Destaca la presencia de acueducto vestibular ensanchado como la anomalía más frecuente, y presenta una relación ampliamente conocida con la ocurrencia de gusher durante la implantación coclear ${ }^{4}$. La incidencia de gusher en nuestro trabajo fue del $4.2 \%$ (12/276), porcentaje que concuerda con lo reportado en la literatura sobre el tema. Hay que tener en cuenta que en nuestro trabajo se distinguió entre oozing y gusher propiamente dicho, como se sugiere en la literatura internacional.

Todos los pacientes con gusher presentaron malformaciones del oído interno visibles en el estudio de imagen prequirúrgico. Sin embargo, debe tenerse en cuenta que esto no es la regla, debido a que no todos los pacientes con anomalías óseas entre el conducto auditivo interno y el espacio subaracnoideo presentan gusher, probablemente por la presencia de bandas fibrosas que sirven de barrera para esta comunicación anómala, pero que son imperceptibles en el estudio de imagen prequirúrgico ${ }^{5}$. Otros autores refieren que también puede deberse a la presencia de una lámina ósea muy delgada, que confunde a los médicos al evaluar el estudio de imagen.

Las opciones de manejo del gusher durante la implantación coclear son diversas y están en continua evolución, y pueden dividirse en no quirúrgicas y quirúrgicas. Entre las opciones no quirúrgicas destacan las que se centran en disminuir el flujo vascular cerebral, con la consecuente disminución de la presión del LCR, e incluyen la posición de Fowler (esta maniobra puede ser de gran ayuda al colocar la guía de electrodos debido a que disminuye considerablemente la fuga de LCR durante unos minutos y brinda así la posibilidad de insertar la guía de electrodos), la hiperventilación, la colocación de un drenaje lumbar

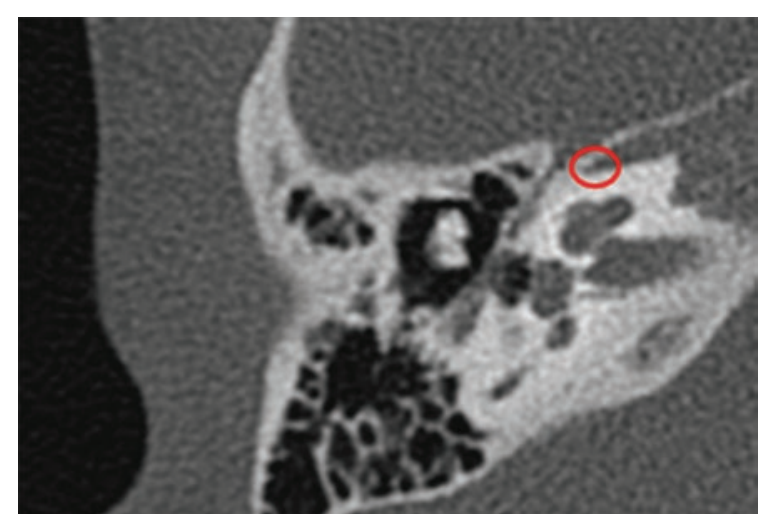

Figura 1. Gusher mínimo. Oído derecho (corte axial) con lámina cribosa ensanchada.

(que suele mantenerse durante 3-5 días) y los medicamentos diuréticos ${ }^{6}$. Las opciones quirúrgicas también son diversas, desde ciertos pasos que deben considerarse durante el procedimiento quirúrgico hasta el diseño de electrodos específicos para el control del gusher. En lo que se refiere al abordaje del oído interno, se recomienda que preferentemente sea a través de la ventana redonda, o en caso de que se realice una cocleostomía, que esta no sea mayor de $1.2 \mathrm{~mm}$ de diámetro, con el fin de que la propia guía de electrodos pueda controlar la fuga en el momento de su inserción. Sin embargo, también hay autores que recomiendan una cocleostomía amplia para que el sellado alrededor de la guía de electrodos sea más fácil ${ }^{6}$. Así mismo, si la visualización es compleja no hay que dudar en retirar el puente o el yunque para un mejor control de la fuga. En cuanto al método de sellado, hay autores que utilizan un fragmento de fascia temporal de $2 \times 2 \mathrm{~mm}$ y realizan un orificio en medio para que pase la guía de electrodos, de modo que cuando la guía se introduzca se selle simultáneamente el acceso al oído interno. No obstante, cabe argumentar que realizar esta maniobra puede limitar la visibilidad del cirujano en el momento de asegurarse de que la colocación es precisa y atraumática. En nuestra institución solemos colocar pequeños fragmentos de fascia temporal alrededor de la guía de electrodos de manera sistemática durante la implantación coclear; esta medida exclusivamente sirvió para un control completo del gusher mínimo que presentaron siete pacientes en nuestra serie (Fig. 1). En cuanto al gusher moderado, una opción es el cierre en dos capas: una de fascia temporal y otra de un fragmento de músculo temporal o de cartílago de trago adherida a la primera capa mediante fibrina. En nuestra serie de pacientes, esta medida fue suficiente para el control de los tres casos de gusher moderado 


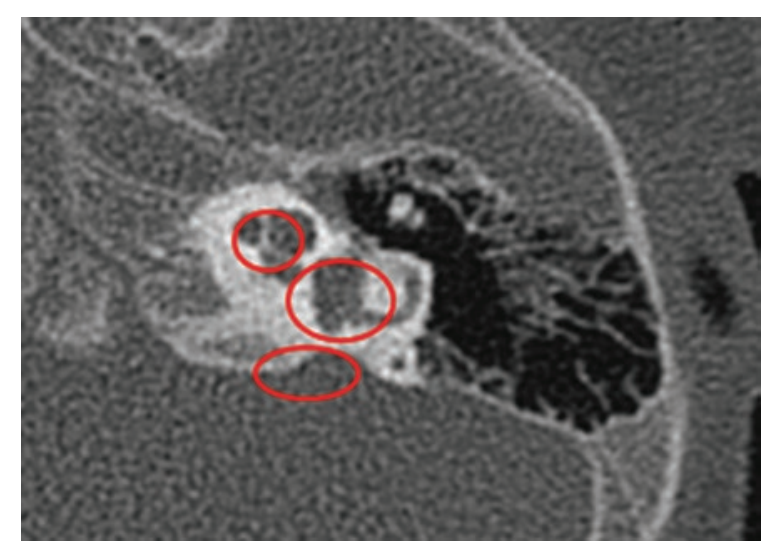

Figura 2. Gusher moderado. Oído izquierdo (corte axial) con las vueltas media y apical de la cóclea que confluyen, vestíbulo dilatado y acueducto vestibular ensanchado (partitura incompleta tipo II).

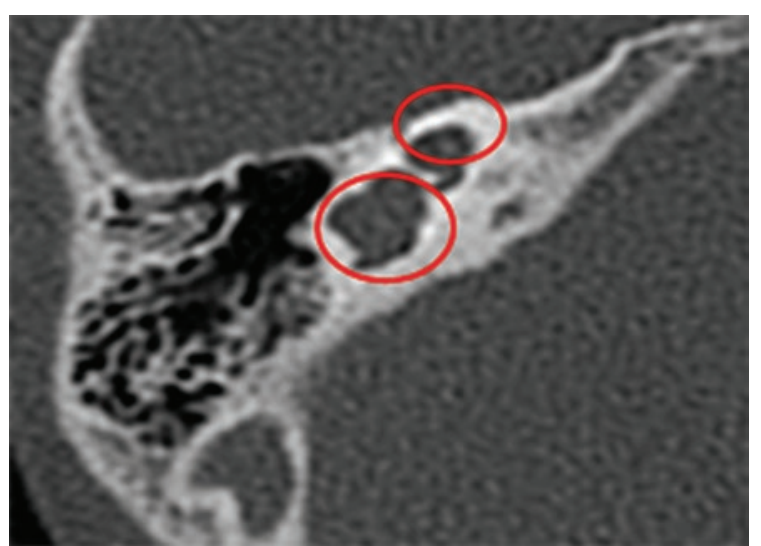

Figura 3. Gusher grave. Oído derecho (corte axial) con la cóclea completamente quística y el vestíbulo muy dilatado (partitura incompleta tipo I).

(Fig. 2). Un gusher grave puede constituir una situación apremiante para el cirujano debido a que puede dificultar de manera notoria la inserción de la guía de electrodos, y en estos casos la literatura recomienda el empaquetamiento del oído medio con cartílago o con material sintético $\left(\right.$ Gelfoam $^{\circledR}$ ) y la obliteración de la trompa de Eustaquio; este fue el manejo que escogimos para los dos pacientes que presentaron gusher grave (Fig. 3).

Es importante destacar que en la actualidad existe una compañía dedicada a la fabricación de implantes cocleares que ha desarrollado implantes con una guía de electrodos específica para gusher. En este tipo de implantes, la porción terminal de la guía de electrodos tiene forma de embudo (de $1.9 \mathrm{~mm}$ de diámetro) en lugar del clásico anillo de silicón, con el fin de prevenir la salida de LCR después de la inserción de la guía de electrodos. Con esta guía se recomienda realizar una cocleostomía de $1.2 \mathrm{~mm}$ de diámetro para que toda la guía de electrodos activos pase y se detenga en el embudo. Es importante destacar que este modelo está disponible en una longitud estándar de $25 \mathrm{~mm}$ y en una longitud corta de $20 \mathrm{~mm}$ para oídos con hipoplasia coclear, y que también cuenta con la ventaja de tener una superficie de estímulo en ambas caras de la guía de electrodos, lo que puede ser muy beneficioso en caso de malformaciones del oído interno en las que no se sabe la localización exacta del tejido neural existente (p. ej., cavidad común) ${ }^{7}$.

Finalmente, otro aspecto de gran relevancia es el pronóstico, tanto en cuanto a la cirugía (morbilidad asociada) como al desempeño del paciente. En relación con el pronóstico quirúrgico, un trabajo realizado por Vashist ${ }^{8}$ encontró que, en un grupo de nueve pacientes que presentaron gusher y que fueron manejados con sellado del sitio de cocleostomía con fascia y periostio, ninguno ameritó medidas extraordinarias para el manejo del gusher, y los autores concluyen que un sellado cuidadoso del sitio de cocleostomía y una vigilancia adecuada son suficientes para prevenir la morbilidad asociada al procedimiento quirúrgico. En lo que se refiere al desempeño del lenguaje de estos pacientes, hay que considerar que un porcentaje importante también presentan comorbilidad neurológica. Farhood, et al. ${ }^{9}$ realizaron una revisión sistemática del desempeño del lenguaje y los hallazgos transoperatorios en pacientes con malformaciones del oído interno, y encontraron un beneficio evidente con el implante coclear para este grupo poblacional. Obtuvieron como hallazgo más relevante que estos pacientes presentaron un desempeño del lenguaje a los 24 meses del implante similar al de los niños con implante sin malformaciones del oído interno, ratificando de esta manera que esta población puede beneficiarse ampliamente de este tipo de tratamiento siempre que se cumplan las consideraciones previas, transoperatorias y posquirúrgicas pertinentes, lo cual coincide con nuestros resultados.

\section{Conclusión}

Es importante enfatizar que debe hacerse una correcta evaluación del estudio de imagen prequirúrgico mediante la toma de medidas específicas, y también determinar la gravedad de cada caso según los hallazgos transoperatorios para poder brindar un manejo individualizado según el grado de gusher.

\section{Conflicto de intereses}

Los autores declaran que no existen conflictos de intereses. 


\section{Financiamiento}

Los autores declaran que no hubo ningún financiamiento.

\section{Responsabilidades éticas}

Protección de personas y animales. Los autores declaran que para esta investigación no se han realizado experimentos en seres humanos ni en animales.

Confidencialidad de los datos. Los autores declaran que han seguido los protocolos de su centro de trabajo sobre la publicación de datos de pacientes.

Derecho a la privacidad y consentimiento informado. Los autores han obtenido el consentimiento informado de los pacientes y/o sujetos referidos en el artículo. Este documento obra en poder del autor de correspondencia.

\section{Bibliografía}

1. Mylanus E, Rotteveel L, Leeuw R. Congenital malformation of the inner ear and pediatric cochlear implantation. Otol Neurotol. 2004;25:308-17.

2. Eftekharian A, Amizadeh M. Cerebrospinal fluid gusher in cochlear implantation. Cochlear Implants Int. 2014:15:179-84.

3. McElveen J, Cunningham C. Cochlear implantation in the congenitally malformed ear. Oper Tech Otolaryngol. 2010;21:243-7.

4. Cabbarzade C, Sennaroglu L. CSF gusher in cochlear implantation: the risk of missing CT evidence of a cochlear base defect in the presence of otherwise normal cochlear anatomy. Cochlear Implants Int. 2015; 16:233-6.

5. Sennaroglu L. Cochlear implantation in inner ear malformations - a review article. Cochlear Implants Int. 2012;11:4-41.

6. Ahmed M, Fawzi M, Samy M. A stepwise algorithm for the management of cerebrospinal fluid gusher during cochlear implantation. The Egyptian Oto-Rino-Laryngological Society. 2015;19-29.

7. Sennaroglu L, Atay G, Munir D. A new cochlear implant electrode with a "cork"-type stopper for inner ear malformations. Auris Nasus Larynx. 2014;41:331-6.

8. Vashist S, Singh S. CSF gusher in cochlear implant surgery - does it affect surgical outcomes? Eur Ann Otorhinolaryngol Head Neck Dis. 2016;133 (Suppl 1):S21-4

9. Farhood Z, Nguyen SA, Miller SC, Holcomb MA, Meyer TA, Rizk HG. Cochlear implantation in inner ear malformations: systematic review of speech perception outcomes and intraoperative findings. Otolaryngol Head Neck Surg. 2017;156:783-93. 